\title{
The different faces of the asthma-COPD overlap syndrome
}

\author{
Dirkje S. Postma and Maarten van den Berge \\ Affiliation: Dept of Pulmonology, University of Groningen, University Medical Center Groningen, GRIAC \\ Institute, Groningen, The Netherlands.
}

Correspondence: D.S. Postma, Dept of Pulmonology, University Medical Center, Hanzeplein 1 AA11, Groningen, The Netherlands. E-mail: d.s.postmađumcg.nl

-

@ERSpublications

A new prospective population-based study in the ERJ makes new strides in understanding the ACOS phenomenon http://ow.ly/PnuaQ

Asthma and chronic obstructive pulmonary disease (COPD) are highly prevalent, chronic, diseases $[1,2]$. Asthma symptoms usually start at a young age, whereas COPD generally becomes symptomatic after the fourth decade of life. It has been recognised in clinical practice and by international asthma and COPD guidelines $[1,2]$ that many people have clinical features compatible with both diseases; the term suggested to describe this is ACOS (asthma-COPD overlap syndrome). However, the underlying mechanisms of ACOS and its appropriate treatment are unclear, particularly since these patients have been excluded from studies determining the pathophysiology and pathology of asthma and COPD, as well as from efficacy studies of respiratory treatments.

When a patient aged around 45 years visits a physician as a result of respiratory symptoms such as wheezing and dyspnoea, it is frequently difficult to determine which disease the patient's symptoms signify. If the patient has a smoking history of 10 pack-years, and airflow limitation with some reversibility, a clinician may tend to label with asthma, yet when the same patient has irreversible airflow limitation the label probably changes to COPD. When irreversible airflow limitation is present, the patient's clinical expression may originate from different pathologies, i.e. from compromised lung function in utero, longstanding and/or severe asthma, or asthma(-like) symptoms in childhood that fade away by an increase in airway diameter from childhood to adulthood and then reoccur due to the natural history of lung function that declines after the age of 25-30 years. It may additionally arise from stunted lung growth in childhood. In the CAMP study, 25\% of mild asthmatics had reduced lung growth over 4-year follow-up, irrespective of treatment [3]. Alternatively, it may be driven by environmental exposures such as smoking, with or without workplace exposure, such as to pesticides and vapours, dust, gas and fumes [4] that induce (small) airway and lung tissue remodelling, by themselves or in addition to the asthma constitution.

The paper in the current issue of the European Respiratory Journal by DE MARCO et al. [5] makes a further step towards understanding the ACOS phenomenon. They performed a prospective population-based study to investigate the differences between asthma, COPD and ACOS. Most studies so far have been performed cross-sectionally and in a limited number of participants, predominantly studying patients with established disease [6, 7], and usually starting with a COPD diagnosis [8-13]. Thus, a diagnosis is probably biased by the doctor's view of asthma and COPD. This is a well-known bias, e.g. with the same level of airway obstruction and smoking, males are predominantly diagnosed with COPD and females with asthma [14].

The novel angle that the study by DE MARCo et al. [5] took was to investigate young adults between 20 and 44 years old, when disease evolution over time is affected by environmental and comorbid factors only to a small extent. They also defined asthma based on a doctor's diagnosis, yet in addition to one of the following: asthma-like symptoms, inhaled respiratory treatment in the past year, airway hyperresponsiveness (AHR)

Received: May 222015 | Accepted: May 222015

Conflict of interest: Disclosures can be found alongside the online version of this article at erj.ersjournals.com

Copyright $\odot$ ERS 2015 
and airflow limitation (forced expiratory volume in $1 \mathrm{~s}$ (FEV1)/forced vital capacity (FVC) less than the lower limit of normal (LLN)) at first visit that had to be normal at the second visit 9 years later, or asthma-like symptoms and AHR in the past year. COPD was present if FEV1/FVC <LLN at the first and second visit in addition to one of the following: COPD-like symptoms, the epidemiological definition of chronic bronchitis, a smoking history of $\geq 10$ pack-years, and occupational exposure to vapours, dust, gas or fumes. They used prebronchodilator spirometric values for their definitions in the first two European Community Respiratory Health Survey (ECRHS) studies, yet elegantly validated this by using postbronchodilator values at a third recent survey. They thus showed the relevance of their findings in four mutually exclusive groups at baseline, i.e. asthma ( $n=941)$, COPD $(n=166), \operatorname{ACOS}(n=218)$ and "healthy" $(n=5659)$.

The main findings the authors report are that individuals with ACOS have risk factors and clinical characteristics comparable with asthma. Their lung function decline over a period of 9 years was similar to the decline in subjects with asthma and significantly lower than in those with COPD. De MARCo et al. [5] made five important observations, as follows.

First, a family history of asthma was equally present in asthma and ACOS, 25 and 26\%, respectively (compared to $18 \%$ in COPD), as were allergen sensitisation, allergic rhinitis and eczema, together hinting at an asthma-type disease. The prevalence of respiratory infections in childhood was highest in asthma and ACOS as well, 17 and 20\% respectively, versus 13\% in COPD. Interestingly, subjects with ACOS reported an earlier age of onset of asthma than subjects with asthma only. In addition, they had a longer disease duration and more frequent asthma exacerbations in the previous year and worse lung function at baseline (FEV1 $85 \%$ predicted) than subjects with asthma (101\% predicted) and COPD (93\% predicted) alone. Together, these findings suggest that early childhood factors, such as infections, or in utero factors, lack of growth in lung function, more severe asthma with frequent exacerbations in earlier life, ongoing airway remodelling with long-standing asthma, or any combination of these, may contribute to ACOS [15, 16]. This may also explain the lower lung function at baseline in ACOS, being even lower than in COPD. This lower lung function was not related to the prevalence of medication use at baseline that was similar in asthma and ACOS. The difference in lung function could still relate to differences in earlier treatment or lack thereof, data that was not available in the study.

Second, the prevalence of lifetime and heavy smoking ( $>15$ pack-years) was quite comparable in "healthy" (55 and 27\%) and asthma (57 and 30\%), a little higher in ACOS (64 and 35\%) and highest in COPD (72 and $52 \%$ ), while exposure to vapours, dust, gas and fumes was significantly higher in COPD (42, 46, 44 and $57 \%$, respectively). This shows the importance of vapours, dust, gas and fume exposure as a risk factor for COPD [4] and of smoking for ACOS and COPD (besides the fact that smoking is harmful anyway). Thus, smoking exposure on top of an "asthma background" (i.e. doctor's diagnosis of asthma, a history of asthma in the family, eczema, rhinitis and allergen sensitisation) may result in airflow limitation, as supported by the observation that mean FEV1 was 101\% predicted in asthma, 85\% predicted in ACOS and 93\% predicted in COPD at baseline (rather high for COPD, but likely compatible with the early phase of the disease).

Third, the authors showed that the "healthy", asthma and ACOS groups have a comparable FEV1 decline over 9 years, their rate of lung function decline being significantly lower than in patients with COPD. The observation that the level of decline is in the normal range in both asthma and ACOS may reflect the time of investigation in younger subjects, predominantly still involving the plateau phase of lung function where there is hardly any change in FEV1. Nevertheless, even though individuals with ACOS had lowest lung function at baseline, they did show a comparable decline with asthma and less so than in COPD, also suggesting a link between ACOS and asthma, while in COPD ongoing effects of smoking and environmental exposures likely will have driven the decline. The latter is speculation, since it has not been reported in the study whether individuals with COPD were persistent smokers during follow-up and those with ACOS not.

Fourth, AHR was present in almost all individuals with ACOS (98\%) and significantly few of those with asthma and COPD (67 and 15\% respectively). This may be partially due to the lower FEV1 values in ACOS, which affect the prevalence of AHR by geometric mechanisms [17], but this impact cannot be large with a mean FEV1 value of $85 \%$ predicted in the ACOS group. However, there is an alternative explanation: several studies have shown that small airway dysfunction at birth contributes to the development of asthma [18], a lower lung function growth in childhood [18], and a more severe AHR and less control of asthma in adulthood [19]. Thus, it could be hypothesised that if small airways were compromised at birth and/or childhood, this might lead to a higher prevalence of AHR in ACOS. Further studies have to assess whether ACOS is a subtype of asthma that develops in association with a compromised small airway function at birth and reduced small airway growth in childhood.

Fifth, it is of interest to note that individuals with persistent airflow limitation (COPD) had significantly more respiratory symptoms compared to the "healthy" group, but were prescribed respiratory treatment 
with almost the same prevalence ( 7 and $5 \%$ respectively), while those with asthma and ACOS had more severe symptoms and were prescribed treatment in more than $70 \%$ of cases. The COPD group had early COPD given their relatively young age, and it is likely that these individuals with persistent airflow limitation do not visit their general practitioner, which is compatible with the fact that they had fewer hospitalisations than those with asthma and ACOS, or they were under-diagnosed and symptoms were assigned to their smoking habits. This may be disadvantageous, when lung function decline progresses at a later stage and cannot be halted.

The advantage of the excellent study of DE MARCo et al. [5] in the current issue of the European Respiratory Journal is the prospective nature. A disadvantage is that diagnoses were still based on preconceptual definitions. Future studies may benefit from a cluster-type analysis, showing in an unbiased way which parameters, both cross-sectionally and longitudinally, dissect the phenotypes that are now called asthma, COPD and ACOS in an age span that the disease is still evolving. This may result in the same outcome, but it may well be that more clusters will emerge and that even ACOS can be subdivided. With the current state of the art, COPD is still defined by FEV1 and FVC. This can be improved [2]! Studies like the ECRHS, important as they are, cannot deliver computed tomography scans or other parameters, like inflammatory and genomic biomarkers. Future studies, then, would preferably also include factors like blood eosinophil counts that, when increased, are risk factors for respiratory symptoms and COPD mortality [20], and reflect a subphenotype of COPD that has a better 1-year response to inhaled corticosteroids with exacerbation frequency but not with the change in FEV1 [21]. It would be of interest to see whether COPD with eosinophilia at adult age fits with development of ACOS over a lifespan. Moreover, the association with treatments will have to be taken into account, since inhaled corticosteroids will be given to many identified patients, and though they usually do not affect lung function decline, a subset of COPD (or is this ACOS?) may respond [22]. Finally, inhaled corticosteroids reduce exacerbations in asthma and may improve remodelling [23], which may affect asthma outcome. The question is whether this also holds in asthma with irreversible airflow limitation. The ideal study would be a longitudinal prospective population study that follows anyone with respiratory symptoms, involving the whole spectrum of future disease. Such a study would then involve robust measurements like spirometry with reversibility, body box, small airway function, AHR, blood cells and constituents, DNA and nasal epithelial cells for (epi)genetic assessments as easy accessible tools separately from bronchoscopies that are more invasive, and computed tomography, to dissect the different subsets of airway and parenchymal diseases. This will be a dream for now, but let us face this important challenge and see how we can develop this study. Let all respiratory pharmaceutical industries, governments and researchers join forces. We throw the gauntlet down... who takes it?

\section{References}

1 Boulet LP, FitzGerald JM, Reddel HK. The revised 2014 GINA strategy report: opportunities for change. Curr Opin Pulm Med 2015; 21: 1-7.

2 Global Strategy for the Diagnosis, Management and Prevention of COPD. Global Initiative for Chronic Obstructive Lung Disease (GOLD), 2015. Available from: www.goldcopd.org

3 Strunk RC, Weiss ST, Yates KP, et al. Mild to moderate asthma affects lung growth in children and adolescents. J Allergy Clin Immunol 2006; 118: 1040-1047.

4 De Jong $\mathrm{K}$, Boezen $\mathrm{H}$, Kromhout $\mathrm{H}$, et al. Pesticides and other occupational exposures are associated with airway obstruction: the LifeLines cohort study. Occup Environ Med 2014; 71: 88-96.

5 De Marco R, Marcon A, Rossi A, et al. Asthma, COPD and overlap syndrome: a longitudinal study in young European adults. Eur Respir J 2015; 46: 671-679.

6 De Marco R, Pesce G, Marcon A, et al. The coexistence of asthma and chronic obstructive pulmonary disease (COPD): prevalence and risk factors in young, middle-aged and elderly people from the general population. PLoS One 2013; 8: e62985.

7 Louie S, Zeki AA, Schivo M, et al. The asthma-chronic obstructive pulmonary disease overlap syndrome: pharmacotherapeutic considerations. Expert Rev Clin Pharmacol 2013; 6: 197-219.

8 Zeki AA, Schivo M, Chan A, et al. The asthma-COPD overlap syndrome: a common clinical problem in the elderly. J Allergy 2011; 2011: 861962.

9 Kauppi P, Kupiainen H, Lindqvist A, et al. Overlap syndrome of asthma and COPD predicts low quality of life. J Asthma 2011; 48: 279-285.

$10 \mathrm{Fu} \mathrm{J}$, Gibson PG, Simpson JL, et al. Longitudinal changes in clinical outcomes in older patients with asthma, COPD and asthma-COPD overlap syndrome. Respiration 2014; 87: 63-74.

11 Hardin M, Cho M, McDonald ML, et al. The clinical and genetic features of COPD-asthma overlap syndrome. EurRespir J 2014; 44: 341-350.

12 Miravitlles M, Soriano JB, Ancochea J, et al. Characterisation of the overlap COPD-asthma phenotype. Focus on physical activity and health status. Respir Med 2013; 107: 1053-1060.

13 Menezes AMB, de Oca MM, Rogelio P, et al. Increased risk of exacerbation and hospitalization in subjects with an overlap phenotype: COPD-asthma. Chest J 2014; 145: 297-304.

14 Han MK, Postma D, Mannino DM, et al. Gender and chronic obstructive pulmonary disease: why it matters. Am J Respir Crit Care Med 2007; 176: 1179-1184.

15 Brown RH, Pearse DB, Pyrgos G, et al. The structural basis of airways hyperresponsiveness in asthma. J Appl Physiol 2006; 101: 30-39. 
16 Postma DS, Bush A, van den Berge M. Risk factors and early origins of chronic obstructive pulmonary disease. Lancet 2014.

17 Postma D, Kerstjens H. Characteristics of airway hyperresponsiveness in asthma and chronic obstructive pulmonary disease. Am J Respir Crit Care Med 1998; 158: S187-S192.

18 Bisgaard H, Jensen SM, Bønnelykke K. Interaction between asthma and lung function growth in early life. Am J Respir Crit Care Med 2012; 185: 1183-1189.

19 Farah CS, King GG, Brown NJ, et al. The role of the small airways in the clinical expression of asthma in adults. J Allergy Clin Immunol 2012; 129: 381-387. e381.

20 Hospers JJ, Schouten JP, Weiss ST, et al. Eosinophilia is associated with increased all-cause mortality after a follow-up of 30years in a general population sample. Epidemiology 2000; 11: 261-268.

21 Pascoe S, Locantore N, Dransfield MT, et al. Blood eosinophil counts, exacerbations, and response to the addition of inhaled fluticasone furoate to vilanterol in patients with chronic obstructive pulmonary disease: a secondary analysis of data from two parallel randomised controlled trials. Lancet Respir Med 2015; 3: 435-442.

22 Lapperre TS, Snoeck-Stroband JB, Gosman MM, et al. Effect of fluticasone with and without salmeterol on pulmonary outcomes in chronic obstructive pulmonary disease: a randomized trial. Ann Intern Med 2009; 151 : 517-527.

23 Sont JK, Willems LN, Bel EH, et al. Clinical control and histopathologic outcome of asthma when using airway hyperresponsiveness as an additional guide to long-term treatment. Am J Respir Crit Care Med 1999; 159: 1043-1051. 